\title{
Régénération naturelle de la forêt tropicale humide de Guyane : étude de la répartition spatiale de Qualea rosea Aublet, Eperua falcata Aublet et Symphonia globulifera Linnaeus f
}

\author{
M Bariteau \\ Station de recherches forestières, BP 709, 97387 Kourou Cedex, Guyane française
}

(Reçu le 9 août 1991; accepté le 11 mars 1992)

\begin{abstract}
Résumé - Des inventaires de régénération naturelle ont été effectués en 1986 et 1988 par échantillonnage systématique au taux de $1 \%$ de la surface et sur 33 espèces de la forêt tropicale humide de Guyane au sein du périmètre sylvicole de Paracou. Cette étude est consacrée à la modélisation de la répartition spatiale de 3 espèces: Qualea rosea Aublet, Eperua falcata Aublet, Symphonia globulifera Linnaeus f. Les principaux paramètres d'abondance et de dispersion sont présentés : densité des semis, précision relative, indice de dispersion. Ce dernier met en évidence l'existence d'agrégats. Deux méthodes mathématiques sont utilisées dans l'étude des structures spatiales (variogrammes et indices non paramétriques). Trois modèles différents apparaissent : agrégats (Qualea rosea Aublet), plaques (Eperua falcata Aublet), paquets (Symphonia globulifera Linnaeus $\mathrm{f}$ ). Ces structures sont en rapport avec la répartition des arbres adultes. Les interactions entre ces deux strates sont décrites, les semenciers pouvant accentuer la mortalité des régénérations à leur proximité. Le milieu exerce également une action sur la répartition des espèces, en particulier la topographie et la fréquence des chablis. Les structures en plaques observées pour Eperua falcata Aublet définissent un type de peuplement à renouvellement rapide, localisé sur les milieux les plus pauvres.
\end{abstract}

sylviculture / régénération naturelle / forêt tropicale / structure spatiale / Guyane française

Summary - Natural regeneration of rainforest in French Guyana: spatial distribution of 3 species, Qualea rosea Aublet, Eperua falcata Aublet, Symphonia globulifera Linnaeus $f$. A natural regeneration survey concerning a systematic sampling of $1 \%$ of the area was carried out from 1986 to 1988 on 33 species from the French Guyana rainforest in the Paracou sylviculture and research area. The present study has focused on spatial distribution modeling of 3 species: Qualea rosea Aublet, Eperua falcata Aublet and Symphonia globulifera Linnaeus $f$. Abundance and dispersion parameters were as follows: seedling density, relative precision, dispersion index. The latter showed the presence of clusters. Two mathematical methods were applied for spatial structure (variograms and non-parametric indices). Three different pattems were found: tight agregates, loose agregates and patches. These structures were related to mature tree distribution. It was found that

\footnotetext{
*Adresse actuelle : INRA, avenue Vivaldi, 84000 Avignon.
} 
interaction between mature trees and young seedlings could lead to partial mortality of seedlings. Species distribution was also related to environment, particularly topography and frequency of gaps. The patch structure observed for Eperua falcata Aublet corresponded to the rapid "turnover rate" focused on the poorest sites.

\section{sylviculture / natural regeneration / tropical forest / spatial patterns / French Guyana}

\section{INTRODUCTION}

La mise au point d'une sylviculture de la forêt tropicale humide nécessite, dans tous les cas, la prise en compte des processus de régénération naturelle. Celle-ci est définie, d'une part, au sens statique, comme l'ensemble des semis et petites tiges existant dans un peuplement, d'autre part, au sens dynamique, comme l'ensemble des processus par lesquels la forêt dense se reproduit naturellement (Rollet, 1969). Cet article est consacré à l'étude de la répartition spatiale pour 3 espèces de la forêt tropicale humide de Guyane, des régénérations naturelles définies au sens statique.

L'exploitation, plus ou moins intensive, d'un massif forestier dans le cadre d'un «aménagement des peuplements naturels" (Maitre, 1982) pose le problème de la reconstitution de l'écosystème et des ressources qu'il contient. Différentes méthodes sylvicoles basées sur la régénération natureile en forêt tropicale humide peuvent être répertoriées :

- celles dont le but principal est d'assister la régénération naturelle après étude des préexistants : Malayan Uniform System en Malaisie par exemple (Wyatt-Smith, 1963);

- celles qui provoquent la régénération naturelle par intervention sur le couvert : Tropical Shelterwood System, par exemple (Rosevear et Lancaster, 1953).

Ces dernières méthodes peuvent être subdivisées en 2 groupes : les variantes intensives qui réclament des interventions directes sur la régénération (enrichissements, dégagements, délianages) et les variantes extensives utilisant des interven- tions indirectes (simple ouverture du couvert) : dans ce dernier cas, la régénération naturelle est simplement induite par des traitements sylvicoles qui visent en premier lieu le peuplement adulte. II faut cependant remarquer le faible nombre d'études quantitatives portant sur la régénération naturelle en forêt tropicale humide, à plus forte raison dans le cadre d'une sylviculture. En effet, les problèmes méthodologiques rencontrés sont très importants dès lors qu'il faut appréhender quantitativement un milieu aussi riche et hétérogène. Les dispositifs sylvicoles ont par nature de grandes surfaces ce qui suppose la mise au point d'échantillonnages appropriés. Des techniques par «sondage» ont été utilisées dans le passé, mais bien souvent, l'objectif sylvicole a été trop réducteur de l'information collectée : c'est le cas dans la méthode Malaise du linear sampling (Barnard, 1950) qui correspond à un échantillonnage de régénération par bandes. Des placeaux carrés contigus (quadrats, en anglais) sont répartis sur des bandes layonnées en forêt. Chaque quadrat est considéré comme «pourvu» de régénération (stocked) si au moins un semis est présent. Le pourcentage de placeaux "pourvus" sert d'indice à la décision sylvicole d'intervenir en coupe dans le peuplement.

La méthodologie d'inventaire des régénérations naturelles au sein du périmètre sylvicole de Paracou, en Guyane française, est basée sur un échantillonnage systématique par placettes circulaires. L'objectif visé est triple :

- estimation quantitative et qualitative des régénérations (densités, hauteurs, croissances...); 
- cartographie descriptive;

- explication des répartitions observées à l'aide de facteurs physiques (climats, sols...) ou biotiques (compétition entre individus et espèces, présence des semenciers...), et évolution dans le temps en fonction des sylvicultures appliquées.

Les principales difficultés méthodologiques proviennent de la grande variabilité dans le mode de répartition des végétaux inventoriés ainsi que la richesse floristique élevée. Cela pose des problèmes quant à la précision a priori des estimations, à la "signification" des images obtenues en cartographie et à la généralisation des relations constatées entre présence de certaines espèces et facteurs du milieu. De là naît le besoin de passer des "cas observés» à des modèles de répartition décrivant les relations dans l'espace entre les individus d'une même espèce ou de plusieurs espèces. Les «structures spatiales» ainsi définies sont des outils de travail indispensables dès lors qu'il faut comparer les résultats d'inventaires répétés dans le temps ou dans l'espace. De plus, la réussite d'une sylviculture basée sur la régénération naturelle, se juge plus par les taux de surface efficacement régénérée que par des estimations de densité de semis à l'hectare. En cela, la connaissance du mode de répartition spatiale des jeunes tiges en forêt naturelle, mise en relation avec les connaissances existantes sur la biologie des espèces (régime de reproduction, dissémination, conditions de germination, interaction entre espèces, compétition entre individus...) pourrait déterminer largement les opérations sylvicoles envisageables à l'avenir dans ce type de forêt en fonction de caractéristiques du peuplement inventorié avant exploitation : intensité des coupes, dévitalisations complémentaires, etc.

Le dispositif d'étude des régénérations naturelles à Paracou, Guyane française, sera décrit dans une première partie, ainsi que les méthodes mathématiques permet- tant d'étudier les structures spatiales. Dans une deuxième partie, les résultats obtenus à partir d'inventaires statistiques seront présentés et discutés : paramètres classiques d'abondance et de dispersion; mise en évidence de structures spatiales à l'aide de trois modèles différents; relations existantes entre les structures observées et la présence d'arbres adultes ainsi qu'avec certains facteurs du milieu.

\section{MATÉRIEL ET MÉTHODES}

Le dispositif sylvicole de Paracou est situé à proximité de la commune de Sinnamary $\left(5^{\circ}\right.$ $15^{\prime} \mathrm{N} ; 52^{\circ} 55^{\prime} \mathrm{O}$ ) en Guyane française (fig 1). Ce site a été choisi comme étant bien représentatif de la forêt actuellement exploitée dans la zone littorale et également à mi-chemin entre les essais de l'Office national des forêts (ONF) déjà existants de Saint-Laurent-du-Maroni et Cayenne-Macouria.

La pluviométrie annuelle est de l'ordre de $3000 \mathrm{~m}$. Sur le plant géologique, les sols de Paracou correspondent aux premiers éléments métamorphiques du "Bouclier guyanais" (migmatites et schistes de la série du Bonidoro).

Le dispositif expérimental pluridisciplinaire de Paracou a vu le jour en 1982, à l'initiative du Centre technique forestier tropical (CTFT). II met en pratique des techniques sylvicoles d' “amélioration du peuplement naturel'. L'objectif est d'accélérer la croissance des tiges d'avenir par des opérations sylvicoles simples et peu coûteuses, au sein d'un peuplement forestier déjà installé (Maitre, 1982) : la régénération naturelle n'est pas "assistée" mais simplement observée. La description de ce dispositif a déjà fait l'objet de plusieurs publications (Schmitt et Bariteau, 1990; Bariteau et al, 1990; CabreraGaillard et Gignoux, 1990).

Le protocole scientifique prévoit un inventaire complet des régénérations naturelles par échantillonnage tous les deux ans : sur chaque placette circulaire du dispositif statistique, 3361 espèces sont inventoriées (tableau I). Ce travail tient compte d'une part, des individus d'une hauteur comprise entre 5 et $150 \mathrm{~cm}$ (petits semis; cercles de rayon $1,17 \mathrm{~m}$ soit un taux de sondage de $1 \%$ ), d'autre part des individus de plus 

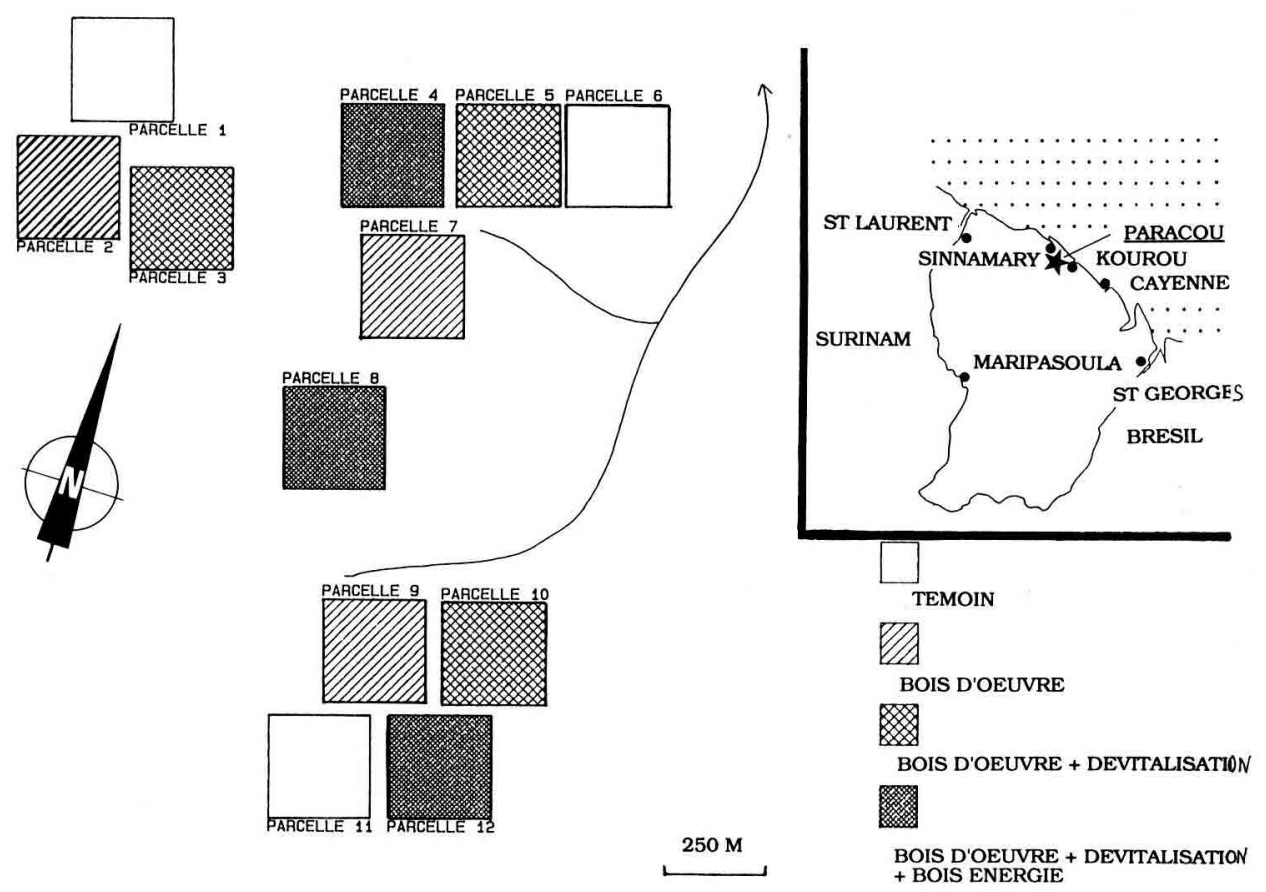

Fig 1. Localisation des parcelles à Paracou.

de $150 \mathrm{~cm}$ de hauteur et de moins de $10 \mathrm{~cm}$ de diamètre (grands semis; cercles de rayon 1,78 $m$ soit un taux de sondage de 2,3\%). Dans chaque parcelle, les points d'inventaire sont répartis tous les $20 \mathrm{~m}$ (fig 2). Les données utilisées dans cet article sont celles des inventaires réalisés en 1986 et 1988. Au cours du premier inventaire (1986), les caractéristiques du milieu sur chaque placeau ont été évaluées par un système de notation : épaisseur de la litière, présence d'un feutrage racinaire situé sous la litière, topographie, concurrence, présence de sous-étage, interception du rayonnement lumineux par le couvert.

Différents paramètres mathématiques ont été calculés à partir de l'inventaire 1988 : densité de semis à l'hectare et précision relative, indice de dispersion.

La densité $D$ des semis à l'hectare pour une espèce est établie à partir de la moyenne des comptages et assortie d'un calcul de précision par la méthode de l'erreur standard. L'indice de dispersion est le rapport de la variance à la moyenne des comptages. II indique le type de répartition spatiale des unités comptées, c'està-dire une dispersion régulière (cas des plantations) pour une valeur significativement inférieure à 1 , aléatoire (conforme à la loi de Poisson) pour un indice égal ou proche de 1, agrégative dans les autres cas. L'indice de dispersion / calculé sur $N$ placeaux peut être testé à l'aide de la valeur $(N-1) \times /$ qui suit un $\chi^{2}$ à $(N-1)$ degrés de liberté. La moyenne par placeau doit être suffisante : suivant les auteurs, supérieure à 1 (Diggle, 1983), à 2 (Dagnélie, 1970) ou 5 (Chessel, 1978). L'approximation normale conduit à une valeur critique de 1,24 au seuil de confiance de $5 \%$ et avec 143 degrés de liberté.

Deux méthodes mathématiques sont utilisées et comparées dans l'étude des répartitions spatiales : les variogrammes étudiés par Matheron dans le cas des variables régionalisées 
Tableau I. Liste des espèces inventoriées à Paracou.

\begin{tabular}{|c|c|c|c|}
\hline Nom commercial & Nom verculaire & Nom scientifique & Famille \\
\hline $\begin{array}{l}\text { Amarante } \\
\text { Amarante } \\
\text { Angelique } \\
\text { Bagasse } \\
\text { Boco } \\
\text { Carapa } \\
\text { Chawari } \\
\text { Cœur dehors } \\
\text { Diaguidia } \\
\text { Bois Saint Jean } \\
\text { Bois Saint Jean } \\
\text { Ebene verte } \\
\text { Wapa } \\
\text { Wapa } \\
\text { Wapa } \\
\text { Gaulette } \\
\text { Gonfolo } \\
\text { Goupi } \\
\text { Grignon } \\
\text { Jacaranda } \\
\text { Kimboto } \\
\text { Mahot cochon } \\
\text { Mahot coton } \\
\text { Mahot noir } \\
\text { Manil montagne } \\
\text { Manil marecage } \\
\text { Parcouri } \\
\text { St Martin jaune } \\
\text { St Martin rouge } \\
\text { Simarouba } \\
\text { Tossopassa } \\
\text { Wacapou } \\
\text { Yayamadou }\end{array}$ & $\begin{array}{l}\text { Dachitan } \\
\text { Papaati } \\
\text { Singapetou } \\
\text { Kaw oudou } \\
\text { Aie oudou } \\
\text { Carapa } \\
\text { Agougagui } \\
\text { Baaka kiabici } \\
\text { Diaguidia } \\
\text { Tobitoutou } \\
\text { Tobitoutou } \\
\text { Guinaati } \\
\text { Bioudou } \\
\text { Bioudou } \\
\text { Bioudou } \\
\text { Koko } \\
\text { Gonfolo kouali } \\
\text { Kopi } \\
\text { Wana } \\
\text { Yachi mambo } \\
\text { Kimboto } \\
\text { Kobe } \\
\text { Caton oudou } \\
\text { Baikaaki } \\
\text { Mataaki } \\
\text { Sabana mataaki } \\
\text { Mongo mataaki } \\
\text { Gueli kiabici } \\
\text { Lebi kiabici } \\
\text { Asoumaripa } \\
\text { Tossopassa } \\
\text { Bounaati } \\
\text { Moulomba }\end{array}$ & $\begin{array}{l}\text { Peltogyne pubescens } \\
\text { Peltogyne venosa } \\
\text { Dicorynia guianensis } \\
\text { Bagassa tiliaefolia } \\
\text { Bocoa prouacensis } \\
\text { Carapa guianensis } \\
\text { Caryocar glabrum } \\
\text { Diplotropis purpurea } \\
\text { Sclerolobium melinonii } \\
\text { Didymopanax morototoni } \\
\text { Schefflera paraensis } \\
\text { Tabebuia serratifolia } \\
\text { Eperua falcata } \\
\text { Eperua grandiflora } \\
\text { Eperua rubiginosa } \\
\text { Licania spp + Parinari spp } \\
\text { Qualea rosea } \\
\text { Goupia glabra } \\
\text { Ocotea Rubra } \\
\text { Jacaranda copaia } \\
\text { Pradosia cohlearia } \\
\text { Sterculia pruriens } \\
\text { Bombax globosum } \\
\text { Escheweilera odora } \\
\text { Moronobea coccinea } \\
\text { Symphonia globulifera } \\
\text { Platonia insignis } \\
\text { Vatairea guianensis } \\
\text { Andira coriacea } \\
\text { Simanuba amara } \\
\text { Iyanthera sagotiana } \\
\text { Vouacapoua americana } \\
\text { Virola spp }\end{array}$ & $\begin{array}{l}\text { Cesalpiniacées } \\
\text { Cesalpiniacées } \\
\text { Cesalpiniacées } \\
\text { Moracées } \\
\text { Cesalpiniacées } \\
\text { Méliacées } \\
\text { Caryocaracées } \\
\text { Cesalpiniacées } \\
\text { Cesalpiniacées } \\
\text { Araliacées } \\
\text { Araliacées } \\
\text { Bignoniacées } \\
\text { Cesalpiniacées } \\
\text { Cesalpiniacées } \\
\text { Cesalpiniacées } \\
\text { Chrysobalanacées } \\
\text { Vochysiacées } \\
\text { Goupiacées } \\
\text { Lauracées } \\
\text { Bignoniacées } \\
\text { Sapotacées } \\
\text { Sterculiacées } \\
\text { Bombacacées } \\
\text { Lecythidiacées } \\
\text { Clusiacées } \\
\text { Clusiacées } \\
\text { Clusiacées } \\
\text { Fabacées } \\
\text { Fabacées } \\
\text { Simaroubacées } \\
\text { Myristicacées } \\
\text { Cesalpiniacées } \\
\text { Myristicacées }\end{array}$ \\
\hline
\end{tabular}

d'une part (Matheron, 1970), les méthodes non paramétriques de la dispersion spatiale des individus d'une espèce, précisées par Chessel (1978) d'autre part.

Les variogrammes ont déjà été employés dans la littérature forestière, pour la mise en évidence de la structure spatiale des peuplements (Bouchon, 1979), de la concurrence entre individus (Bachacou et Decourt, 1976), des relations entre la faune et la végétation (Hladik, 1979), etc.

Une variable est dite régionalisée lorsque les valeurs observées dépendent de la position dans l'espace des points d'observation. La forme du variogramme, à l'origine, est en liaison avec la nature du phénomène observé (voir fig 3). En pratique, nous distinguerons les cas suivants :

- variogramme plat : "pas de structure" ou structure de taille inférieure à la dimension de la maille;

- variogramme décroissant : les valeurs à petite échelle sont plus élevées que la variance; structure de taille inférieure ou égale à la maille de l'échantillonnage. II y a dans ce cas une forte hétérogénéité entre relevés voisins (voir plus loin la notion de "contagion");

- variogramme croissant : les valeurs à petite échelle sont moins élevées que la variance; structure de taille supérieure à la maille. Un pa- 


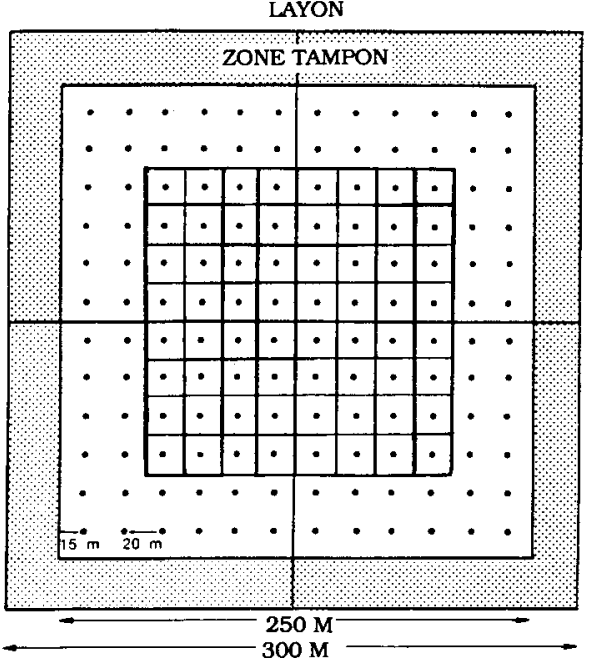

Fig 2. Schéma du dispositif et découpage en 8 x 8 placettes pour le test de Greig-Smith. Chaque point correspond à l'emplacement d'une placette circulaire (en tout $12 \times 12=144$ placeaux par parcelle).

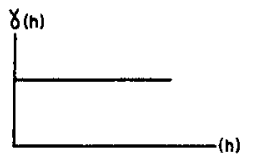

Voriogramme plat "pas de structure"

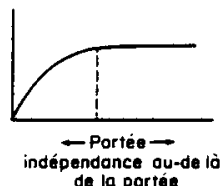

de la portée

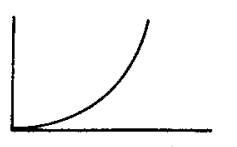

Structure très régutière

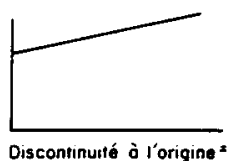

structure très irrégulière (du moins d l échello considéráe)
Fig 3. Différents comportements de variogrammes expérimentaux (d'après Bachacou et Decourt, 1976, p 181).

lier est atteint pour la valeur de la "portée" ce qui donne une indication sur la taille de la structure, puis les valeurs oscillent autour de $s^{2}$, la variance de l'échantillon. Le palier théorique correspond à un maximum pour le variogramme expérimental. Au-delà de ce maximum, les fluctuations observées ne sont plus interprétables.

Les tests non paramétriques à taille de blocs croissante utilisent le regroupement des unités de comptage en blocs contigus. Dans le cas où une grille de comptage comporte $2^{b}$ unités élémentaires, on réalise $b$ partitions de la grille, chaque bloc de taille $k$ étant le regroupement de deux blocs contigus de taille $k / 2$. Dans le cas de Paracou, on prendra un périmètre central de $8 \times$ 8 placettes (voir fig 2). Le test le plus classique est celui de Greig-Smith (Greig-Smith, 1952 in Upton et Fingleton, 1985).

Chessel (1978) utilise 6 paramètres statistiques, à 3 niveaux différents :

- la variabilité totale entre blocs de même taille : décrite à l'aide de l'indice de dispersion classique et de lindice de dispersion non paramétrique;

- la variabilité locale (entre unités dans un même bloc) : décrite par l'indice de dispersion locale et l'indice des cases vides;

- la variabilité à échelle fixée (variabilité entre couples de blocs voisins) : décrite par l'indice de contagion vraie et la variance locale.

Chessel propose une typologie des structures et un comportement standard des indices dans chaque cas (voir tableau II). Les principaux cas rencontrés en forêt font appel, pour être déterminés, en plus de l'indice de Greig-Smith, à l'indice de dispersion non paramétrique et l'indice de contagion vraie (plaques, agrégats et paquets). Nous utiliserons 3 indices dans cet article :

- l'indice de dispersion non paramétrique mesure de façon classique une hétérogénéité globale entre blocs de taille donnée dans un échantillon systématique en présence-absence. Les unités sont comptées + ou - selon que le contenu est inférieur ou supérieur à la médiane observée pour la totalité de la parcelle;

- l'indice de Greig-Smith teste l'hétérogénéité entre couples de blocs voisins, à une échelle fixée par référence à l'hétérogénéité entre blocs de taille 1 voisins. La méthode conduit à l'emploi d'un test $F$;

- l'indice de contagion vraie mesure également l'hétérogénéité entre couples de blocs voisins et complète l'indice de Greig-Smith en permettant un test au niveau de la case élémentaire (caractérisation des dispersions en paquets en cas d'indice de contagion élevé : cela correspond au cas des variogrammes décroissants à l'origine). 
Tableau II. Structures-modèles décrites par Chessel et leurs caractéristiques (d'après Chessel 1978, p 119).

Modèles Caractéristiques

Poissonien

Les tests sont en majorité non significatifs. La meilleure caractérisation est un indice $I_{D}$ voisin de $\mathrm{I}$ aux différentes tailles de bloc. Exemples : plantes du milieu désertique, bactéries au laboratoire.

Dispersion en paquets Dispersion forte. Courbe de dispersion non paramétrique nulle. Dispersion locale et contagion vraie très significative aux petites tailles de bloc. Exemples : insectes par plantes.

Dispersion poissonnienne à variations de densité

Dispersion faible. Courbe de dispersion non paramétrique croissante Dispersion locale non significative pour différentes tailles de bloc. Contagion vraie croissante. Exemples : vers blancs, plantes steppiques.

Dispersion en agrégats

Courbe de dispersion non paramétrique et courbe de contagion vraie en cloche avec maximum net. Exemple : bouquets en milieu forestier.

Dispersion en plaques

Courbe de dispersion non paramétrique et test de Greig-Smith très significatifs dans les grandes tailles de bloc. Figures en général explicites. Exemple : forêt.

Structures à deux échelles d'hétérogénéité

Courbe de contagion vraie et de dispersion à deux maxima.

Test de Mead ou des suites I dans 4 significatits à deux tailles de bloc. Exemples : plantes steppiques, prairies.

Structures complexes

Reprendre des analyses partielles soit en changeant la taille des cases soit en se limitant à une partie de la grille. Exemples : dispersion localement régulière à densité perturbée, dispersion en paquets de densité variable, dispersion à trous...

Sur le dispositif de Paracou, mis à part pour le calcul de l'indice de Greig-Smith (voir plus haut), le découpage des parcelles en blocs successifs est fait dans l'ordre suivant (le premier chiffre indique le nombre de placettes agrégées sur la ligne, le suivant sur la colonne) :

$(1 \times 1),(2 \times 1),(2 \times 2),(3 \times 2),(3 \times 3),(6 \times 3)$, $(6 \times 6),(12 \times 6),(12 \times 12)$

Afin de pouvoir établir les comparaisons graphiques entre indices et de façon standard, par espèce et par parcelle, les valeurs du variogramme sont normalisées par la valeur de la variance, et l'indice de Greig-Smith est divisé par la valeur du $F$ théorique servant au test puis comparé à 1 .

Sur un second graphique sont portés l'indice de dispersion non paramétrique et l'indice de contagion vraie. Les valeurs critiques, pour une signification au seuil de $5 \%$, sont indiquées (respectivement 3 et 2 ).

Pour obtenir une même échelle de comparaison pour la taille des structures, sont portées en abscisse des graphiques : la valeur de $h$ pour les variogrammes (distance entre points servant au calcul du variogramme expérimental), et la dimension maximale entre deux points appartenant aux blocs pour les méthodes non paramétriques (diagonale des carrés ou des rectangles formés par les blocs). Par exemple, pour un regroupement $2 \times 2$, la distance de référence est :

$$
40 \times \sqrt{2}=56,57 \mathrm{~m}
$$

Pour l'approche des structures spatiales, nous étudierons les résultats de l'inventaire de 1988 dans les 3 parcelles témoins non touchées par l'exploitation (parcelles 1, 6, 11) en limitant à trois espèces correspondant à des modèles dif- 
férents : Qualea rosea, Eperua falcata, Symphonia globulifera.

Pour ces 3 espèces, et dans le but d'établir des relations entre la régénération naturelle et le peuplement adulte, un histogramme a été construit avec le nombre de présences observées dans l'inventaire 1986, avant exploitation forestière, (pour 1728 relevés, c'est-à-dire l'ensemble des 12 parcelies) en fonction de la distance au plus proche adulte (diamètre minimal $30 \mathrm{~cm}$ ), par classes de $5 \mathrm{~m}$ : classe 5 pour les distances de 0 à $5 \mathrm{~m}$ inclus, etc jusqu'à la classe 35 pour les distances comprises de 30 à $35 \mathrm{~m}$ inclus). Cette méthode est globale et ne permet pas, en principe, d'affecter chaque pésence à un "semencier" déterminé. Elle permet cependant d'évaluer les relations entre strates et l'évolution des régénérations dans le temps par la comparaison des résultats entre "petits semis" et "grands semis". Le premier inventaire, réalisé en 1986 n'avait pas tenu compte des effectifs réels de plantules mais d'une estimation basée sur les critères suivants : note 0 , pas de semis; note 1, 1 ou 2 semis; note 2, 3 à 9 semis (inclus); note 3, 10 semis et plus. Les histogrammes présentés sont contruits à partir de cette notation de présence-abondance.

La mise en évidence de structures spatiales par la voie mathématique est complexe. De nombreux calculs peuvent être évités par cartographie des résultats, en faisant figurer par des niveaux de couleurs ou de grisés l'intensité des phénomènes observés. À Paracou, des cartes sont obtenues pour chaque espèce comptée, sur chaque parcelle et à chacune des dates dinventaire. La mise en évidence de la dynamique des "taches de semis" pourra se faire, dans les cas les plus simples, en superposant les cartes obtenues aux différents stades d'évolution des peuplements. Un lissage améliore encore la lisibilité : la carte brute figure pour un carré élémentaire $(20 \mathrm{~m} \times 20 \mathrm{~m})$, la densité des semis relevée au point central (piquet d'inventaire); la carte lissée établit pour la même zone, une densité moyenne calculée sur le voisinage (fig 4). La cartographie sera utilisée ici comme un outil complémentaire, indispensable dans certains cas pour l'interprétation biologique (la connaissance du milieu est à ce stade indispensable : relief, caractéristiques du peuplement, etc).

Afin de préciser, espèce par espèce, les liaisons entre présence de régénération et facteurs du milieu, nous avons calculé pour chaque classe d'un facteur écologique donné, l'indice de fréquence corrigée (voir exemple tableau III). Pour cela, il est nécessaire de diviser les fréquences relatives de présence (ou absence) de l'espèce dans chacune des classes du paramètre étudié, par la fréquence relative de présence (ou absence) de l'espèce dans l'ensemble des relevés (Daget et al, 1974). La représentation graphique en ufuseaux" permet d'apprécier le comportement global des espèces.

\section{RÉSULTATS ET DISCUSSION}

Dans ce chapitre, sont simultanément présentés et discutés 4 ensembles de résultats envisageables à partir de l'échantillonnage systématique effectué à Paracou.

\section{Paramètres d'abondance et de dispersion}

Le tableau IV présente les principaux paramètres classiques pour les espèces inventoriées. Beaucoup d'espèces sont faiblement présentes en régénération dans la forêt naturelle et ne peuvent être décrites correctement par ce type d'inventaire statistique : c'est le cas de Goupia glabra (le goupi) qui ne devient très abondant qu'après exploitation. L'échantillonnage systématique a alors l'avantage de réaliser un «point zéro» avant traitement. Dans

Tableau III. Exemple de calcul de l'indice de fréquence corrigée. Indices de fréquence corrigée : présence de l'espèce dans la classe du facteur $1:(c \times M) /(a+c) \times(c+d)$; présence de l'espèce dans la classe du facteur $2:(d \times M) /(b+$ d) $x(c+d)$.

Facteur 1 Facteur 2 Total

$\begin{array}{lccc}\text { Absence de l'espèce } & a & b & a+b \\ \text { Présence de l'espèce } & c & d & c+d \\ \text { Total } & a+c & b+d & N\end{array}$




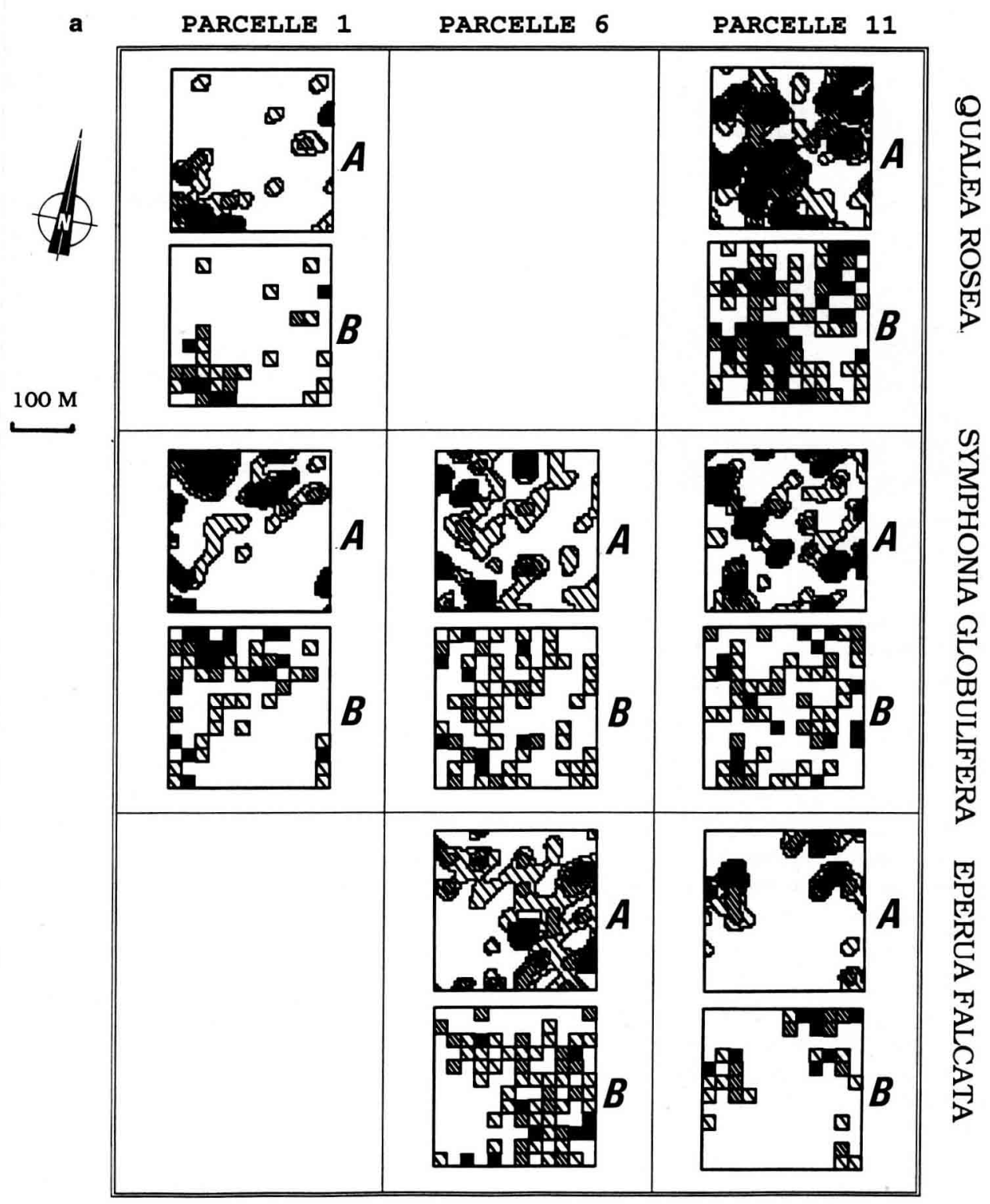

$\mathrm{A}=$ carte lissée; $\mathrm{B}=$ carte brute

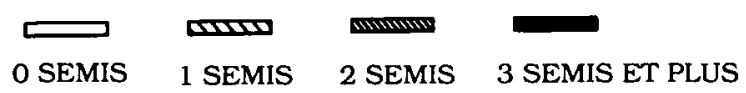

Fig 4. a. Cartographies des régénérations (petits semis, inventaire 1988). 
b

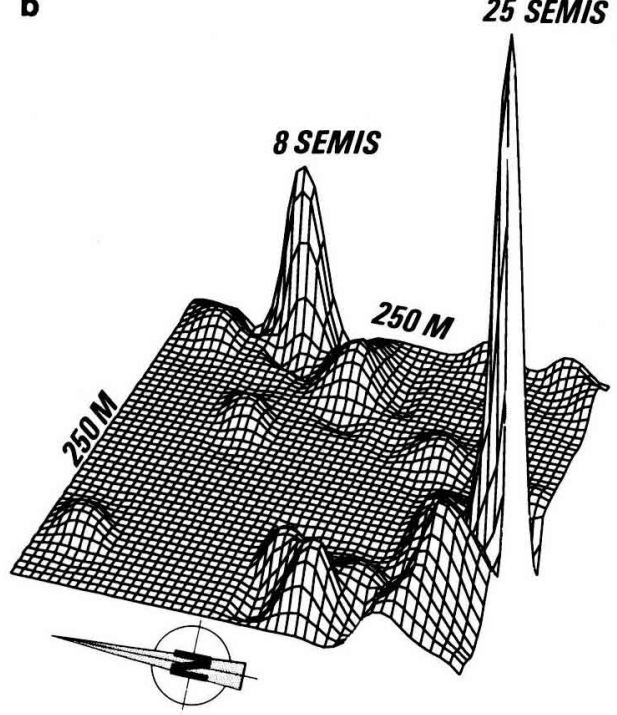

Fig 4. b. Cartographie 3 dimensions (parcelle 1 Qualea rosea).

d'autres cas, les semis sont présents par paquets de petites tailles qui échappent en partie aux "mailles du filet». C'est le cas de Vouacapoua americana (wacapou). La présence de l'espèce risque alors d'être sous-estimée dans le calcul des paramètres mais l'étude des structures mettra le phénomène en évidence.

Dans l'étude des structures spatiales, nous nous limiterons à 3 espèces, bien déterminées sur le plan botanique, bien représentées en régénération et dans le peuplement, et dont la dynamique propre pourra senvir de modèle pour l'étude d'autres essences moins abondantes : Qualea rosea (gonfolo), Eperua falcata (wapa) et Symphonia globulifera (manil marécage). Contrairement à l'ensemble des «gaulettes" (Licania spp et Parinari spp) et des «mahots" (Eschweilera spp) présents uniformément dans le peuplement adulte, ces 3 espèces sont caractéri- sées par des abondances très variables dans l'espace, allant jusqu'à l'absence complète par zones. Les densités moyennes pour ces 3 essences, lorsqu'elles sont présentes, vont de 1000 à 4000 semis à l'hectare. Les indices de dispersion sont d'ordre très différent dans les 3 cas : en moyenne de 2,6 pour Eperua falcata, 6,6 pour Qualea rosea et 16,2 pour Symphonia globulifera. On constate, pour les 3 espèces étudiées et lorsque le calcul est possible, que les semis sont toujours sous-dispersés (répartis en agrégats).

\section{Étude des structures spatiales}

Les résultats sont présentés graphiquement sur les figures 5 à 7 .

\section{Qualea rosea}

Dans la parcelle 1, l'indice de dispersion non paramétrique indique une double échelle d'hétérogénéité correspondant aux distances 85 et $170 \mathrm{~m}$ (regroupements $3 \times$ 3 et $6 \times 6$ ). L'indice de Greig-Smith confirme l'existence d'agrégats de taille supérieure à $110 \mathrm{~m}$. Le variogramme OuestEst révèle 2 échelles d'agrégation avec "portées" à 60 et $120 \mathrm{~m}$. Le variogramme Nord-Sud montre un net phénomène de "dérive» propre à un gradient continu de densité des semis. L'image «lissée» révèle $1 / 4$ de parcelle régénérée avec un noyau central et quelques "grains» périphériques.

Dans la parcelle 11 , les variogrammes sont pratiquement plats ce qui pourrait faire croire, a priori, à une indépendance entre relevés. Les indices non paramétriques montrent une double échelle d'agrégation à 40 et $135 \mathrm{~m}$. L'indice de GreigSmith confirme l'existence d'agrégats de taille supérieure à $110 \mathrm{~m}$. L'image «lissée» montre une parcelle remplie de façon homogène avec des zones plus denses cor- 
respondant aux échelles d'hétérogénéité relevées par le calcul.

\section{Eperua falcata}

Les indices non paramétriques sont très explicites pour la parcelle 6 : l'indice de contagion vraie et l'indice de dispersion non para- métrique croissent avec des valeurs élevées aux grandes tailles de bloc. II s'agit d'une structure en plaque, confirmée par l'image. Les variogrammes oscillent autour de zéro, le variogramme moyen étant pratiquement plat. Les valeurs du test de GreigSmith tendent vers les seuils significatifs aux grandes tailles de blocs.

Tableau IV. Principaux paramètres d'abondance et de dispersion (parcelles témoin, inventaire 1988). $D i=$ densité de régénérations dans la parcelle $i$ (nombre de semis/ha). Pri $=$ précision relative sur $\mathrm{D} i$ (en \%). li : indice de dispersion dans la parcelle $i .16$ semis/ha correspond à 1 semis inventorié par parcelle.

\begin{tabular}{|c|c|c|c|c|c|c|c|c|c|}
\hline Espèce & D1 & $\operatorname{Pr} 1$ & 11 & D6 & Pr6 & 16 & $D 11$ & Pr11 & 111 \\
\hline Andira coriacea & 0 & . & . & 0 & . & . & 0 & & . \\
\hline Bagassa tiliaefolia & 0 & . & . & 0 & & . & 0 & & . \\
\hline Bocoa prouacensis & 976 & 18,26 & 2,03 & 352 & 25,25 & 1,40 & 1616 & 13,19 & 1,76 \\
\hline Bombax globosum & 0 & & & 0 & & & 32 & 70,46 & 0.99 \\
\hline Carapa guianensis & 224 & 29,24 & 1,20 & 80 & 44,09 & 0.97 & 160 & 30,61 & 0,94 \\
\hline Caryocar glabrum & 0 & & & 0 & & . & 0 & & \\
\hline Dicorynia guianensis & 160 & 36,60 & 1,34 & 240 & 37,51 & 2,11 & 240 & 36,30 & 1,98 \\
\hline Didymopanax morototoni & 16 & $\ldots$ & --- & 0 & & & 0 & & . \\
\hline Diplotropis purpurea & 0 & . & 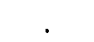 & 96 & 40,10 & 0,96 & 96 & 84,87 & 4,32 \\
\hline Eperua falcata & 0 & & & 1744 & 14,28 & 2,22 & 1040 & 21,48 & 3,00 \\
\hline Eperua grandiflora & 704 & 84,62 & 31,50 & 3136 & 23,64 & 10,95 & 3104 & 20,94 & 8,50 \\
\hline Eperua rubiginosa & 0 & & & 0 & & & 0 & & \\
\hline Eschweilera odora & 9424 & 21,87 & 28,16 & 6528 & 12,22 & 6,09 & 3200 & 11,33 & 2,57 \\
\hline Goupia glabra & 16 & -.- & $\ldots$ & 16 & $\ldots$ & --- & 32 & 70,46 & 0,99 \\
\hline Iryanthera sagotiana & 1872 & 12,13 & 1,72 & 976 & 14,82 & 1,34 & 352 & 21,70 & 1,04 \\
\hline Jacaranda copaia & 16 & --- & $\ldots$ & 48 & 57,33 & 0,99 & 0 & & \\
\hline Licania + Parinari spp & 3872 & 12,39 & 3,72 & 3152 & 9,39 & 1,74 & 3120 & 9,25 & 1,67 \\
\hline Moronobea coccinea & 0 & & & 256 & 29,57 & 1,40 & 208 & 28,70 & 1,07 \\
\hline Ocotea rubra & 144 & 39,32 & 1,39 & 16 & -- & 1,00 & 112 & 36,99 & 0,96 \\
\hline Peltogyne pubescens & 16 & --- & --- & 32 & 70,46 & 0,99 & 0 & . & . \\
\hline Peltogyne pubescens & 0 & & & 0 & & . & 0 & . & \\
\hline Platonia insignis & 32 & 70,46 & 0,99 & 16 & $\ldots$ & --. & 16 & --- & 1,00 \\
\hline Pradosia cochlearia & 912 & 33,61 & 6,44 & 2880 & 17,78 & 5,69 & 3680 & 20,87 & 10,02 \\
\hline Qualea rosea & 1232 & 35,69 & 9,81 & 0 & . & . & 4128 & 11,42 & 3,36 \\
\hline Schefflera paraensis & 0 & & & 0 & & & 0 & & \\
\hline Sclerolobium melinonii & 128 & 55,47 & 2,46 & 288 & 31,41 & 1,78 & 2224 & 32,77 & 14,93 \\
\hline Simaruba amara & 16 & -.- & -- & 16 & -.- & --- & 64 & 60,88 & 1,48 \\
\hline Sterculia pruriens & 1344 & 56,04 & 26,39 & 480 & 31,33 & 2,94 & 224 & 35,58 & 1,77 \\
\hline Symphonia globulifera & 2256 & 19,66 & 5,45 & 3328 & 40,29 & 33,76 & 2560 & 24,36 & 9,49 \\
\hline Tabebuia serratifolia & 0 & . & . & 0 & . & . & 0 & . & . \\
\hline Vatairea guianensis & 0 & & & 0 & & . & 0 & . & . \\
\hline Virola sp pl & 592 & 27,32 & 2,76 & 208 & 34,37 & 1,54 & 416 & 25,40 & 1,68 \\
\hline Vouacapoua americana & 192 & 30,15 & 1,09 & 208 & 36,06 & 1,69 & $\$ 12$ & 54,88 & 2,11 \\
\hline
\end{tabular}


PARCBLLE 01 gUALEA ROBEA

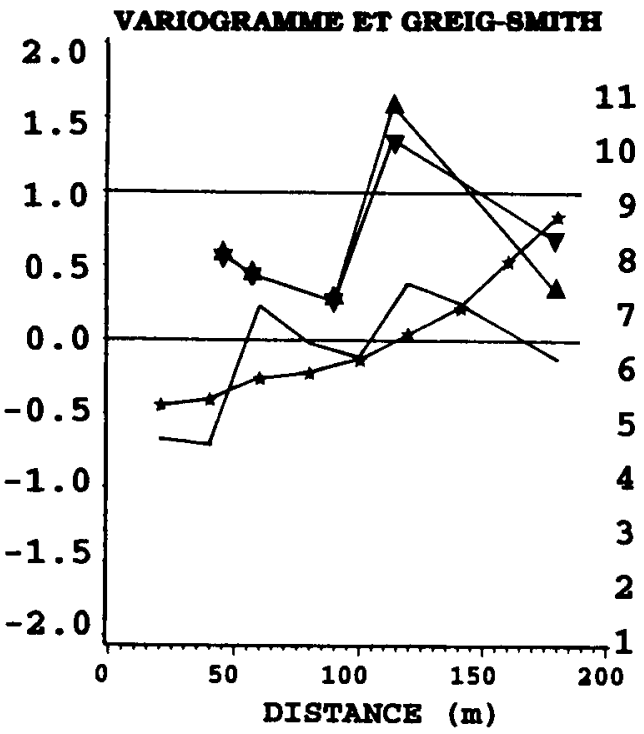

INDICES NON PARAMTERIgUES

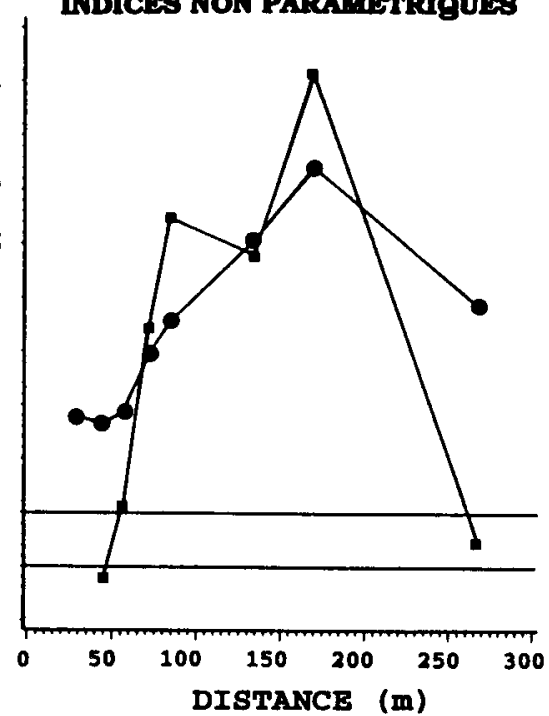

PARCELLE 01 SYMPFONL GLOBULTERA VARIOGRAMATE ET GREIG-8MTTE INDICES NON PARAMETRIgUES
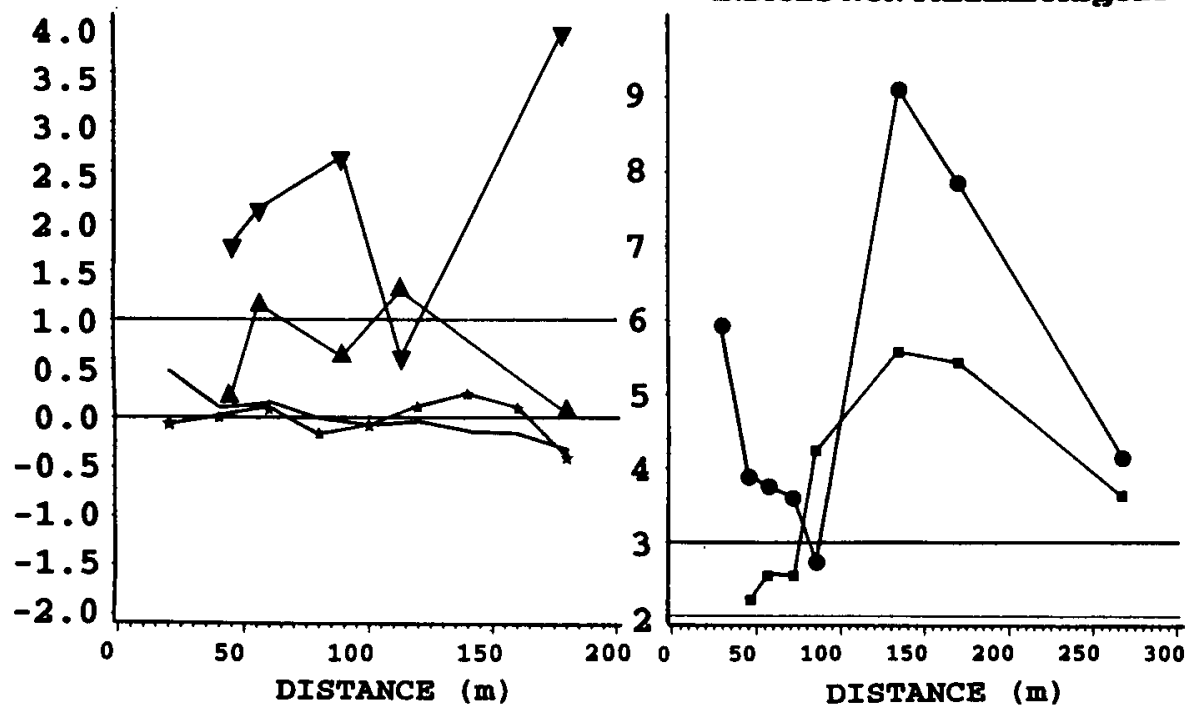

Fig 5. Variogrammes et indices non paramétriques, parcelle 1. 
PARCELUE 06 EPERUA FALCATA VARIOGRAMAE ET GRMIG-8MTTH IDDICES NON PARAMTTRIGUES

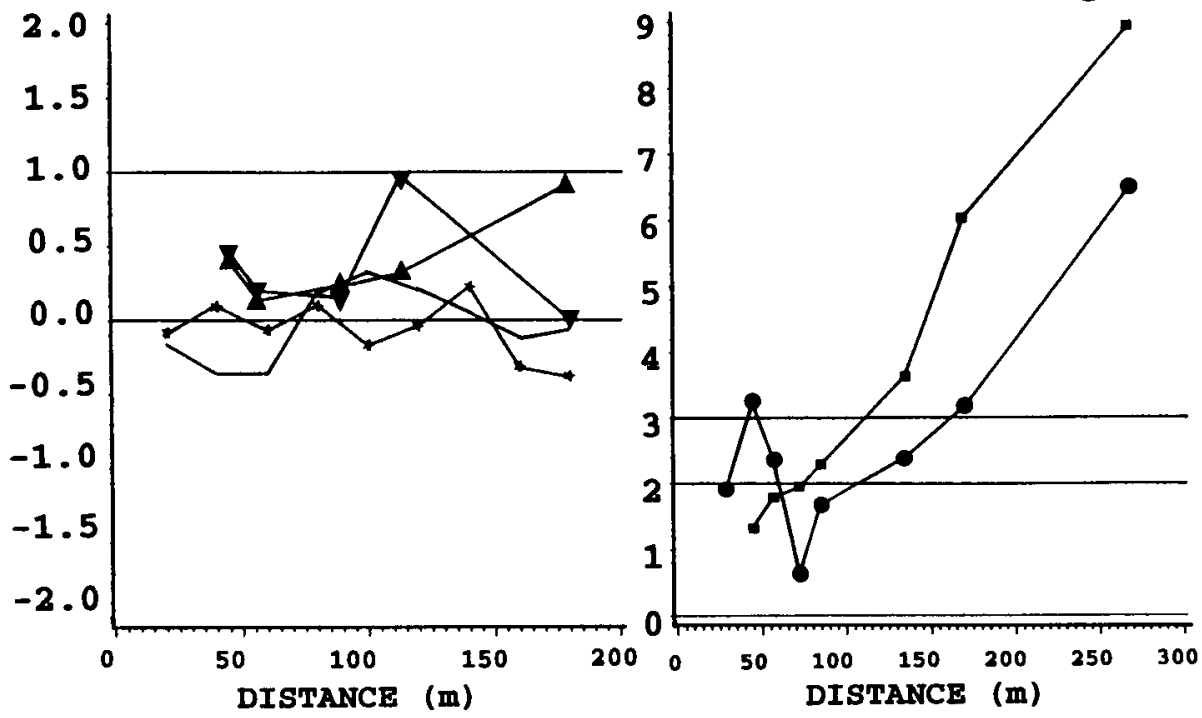

PARCELLE 06 STMPHONA GLOBULTERA

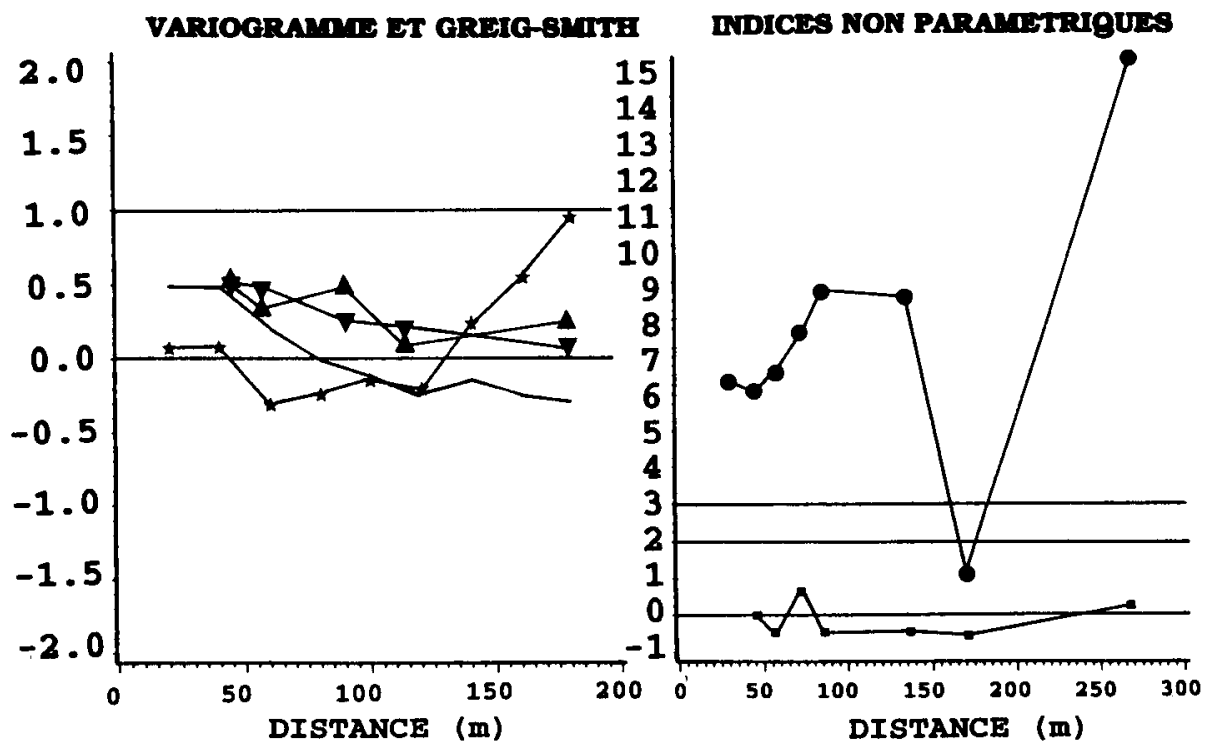

Fig 6. Variogrammes et indices non paramétriques, parcelle 6. 

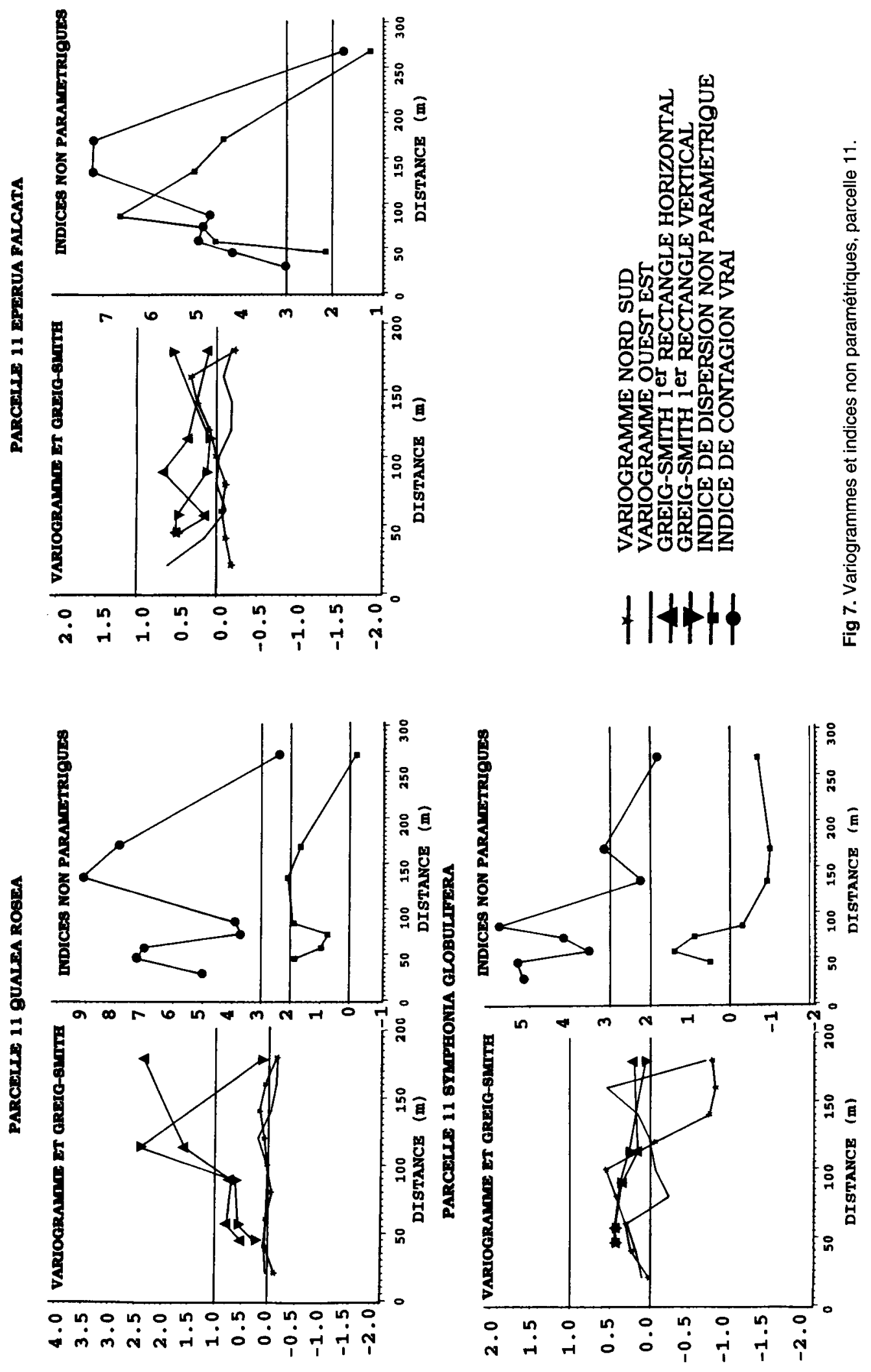
La parcelle 11 montre l'existence d'agrégats de taille 75 et $135 \mathrm{~m}$. Le variogramme Nord-Sud atteint la «portée» entre 140 et $160 \mathrm{~m}$ mais les valeurs ne sont plus significatives à cette échelle.

La cartographie de l'ensemble des parcelles en 1986 (avant exploitation) met en évidence l'existence de grandes plaques de régénération sur lesquelles sont assises les parcelles (voir fig 8). La partie Nord-Est du dispositif est localisée dans une zone très riche en Eperua falcata alors que les parcelles 1,2 et 3 en sont pratiquement dépourvues. La structure au sein de la plaque de la parcelle 6 a été précisée par le calcul de l'indice de dispersion et des indices non paramétriques dans le carré Sud-Est (de taille $125 \mathrm{~m} \times 125 \mathrm{~m}$ ). Les indices sont non significatifs au seuil de $5 \%$, aux différentes tailles de blocs, sauf la dispersion classique I qui indique une aggrégation pour les regroupements $1 \times 1$ (maille élémentaire) et $2 \times 1$. Cela correspond à une distance maximale de $40 \mathrm{~m}$.

\section{Symphonia globulifera}

La dispersion de Symphonia globulifera est typiquement "en paquets» : indices de dispersion non paramétriques en général non significatifs et indices de contagion vraie élevés aux petites tailles de bloc. Les variogrammes sont globalement décroissants ce qui traduit une forte hétérogénéité entre cases élémentaires. La parcelle 1 confirme cette analyse aux petites tailles de blocs. De plus, une structure en agrégat est nette à la dimension $135 \mathrm{~m}$. En fait le Sud-Est de la parcelle est vide : relief accentué avec drainage latéral fort, milieu pour lequel le «manil marécage» n'est pas adapté. La taille des paquets ne peut être déterminée par le modèle car elle correspond à un ordre de grandeur inférieur ou égal à la dimension de la maille. Des précisions sont apportées par l'étude des relations entre semis et arbres adultes.

\section{Présence de semis et proximité des arbres adultes}

L'approche biologique doit permettre de relier l'existence des structures, mises en évidence par le calcul, avec la dynamique de régénération propre à chaque espèce. La démarche la plus naturelle consiste à mettre en rapport la présence des semis avec la proximité des arbres adultes ainsi qu'avec les principaux facteurs du milieu observables.

\section{Qualea rosea}

Près de $70 \%$ des "petits semis" se trouvent à moins de $15 \mathrm{~m}$ d'un arbre adulte (fig 9). Les taches de régénération sont donc très liées à la présence de semenciers. Les différentes échelles d'agrégation déjà notées correspondent à ces «bouquets" (120 à $170 \mathrm{~m}$ ) alors que les agrégats de taille inférieure (40 à $85 \mathrm{~m}$ ) sont vraisemblablement à l'échelle d'un ou de deux semenciers à la proximité desquels la densité de semis est encore supérieure. L'histogramme des grands semis a une forme comparable ce qui semble prouver que la sélection au sein des régénérations s'exerce de façon homogène mais aussi de façon sévère car les notes d'abondance 3 (plus de 9 semis par placeau) ne sont plus représentées. Le taux de mortalité des semis calculé sur 22 mois, est de 23\% (Bariteau et Geoffroy, 1989). La classe $5 \mathrm{~m}$ ne représente que $15 \%$ des effectifs «petits semis». Ce phénomène s'observe sur la plupart des espèces et s'explique vraisemblablement par le fait que les graines sont plus abondantes sur la partie périphérique du houppier. On trouve donc en moyenne moins de semis près du tronc d'un arbre adulte qu'à quelques mètres de celui-ci. Les effectifs dispersés entre 30 et $35 \mathrm{~m}$ correspondent à plus de $6 \%$ en petits semis et $9 \%$ en grands semis : l'espèce tend vrai- 

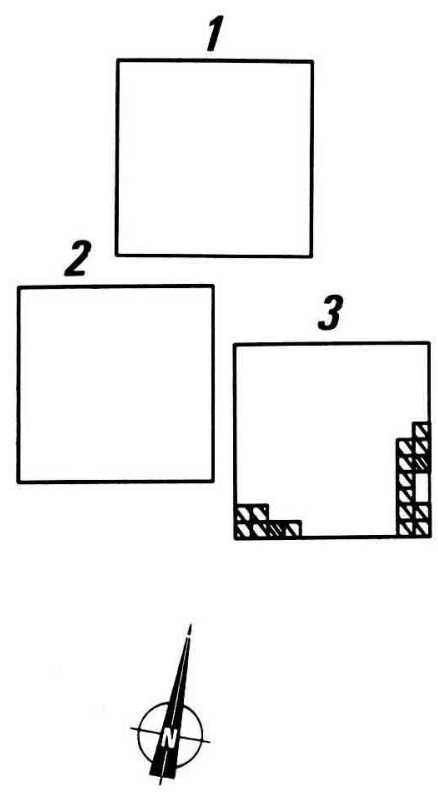

$100 \mathrm{M}$
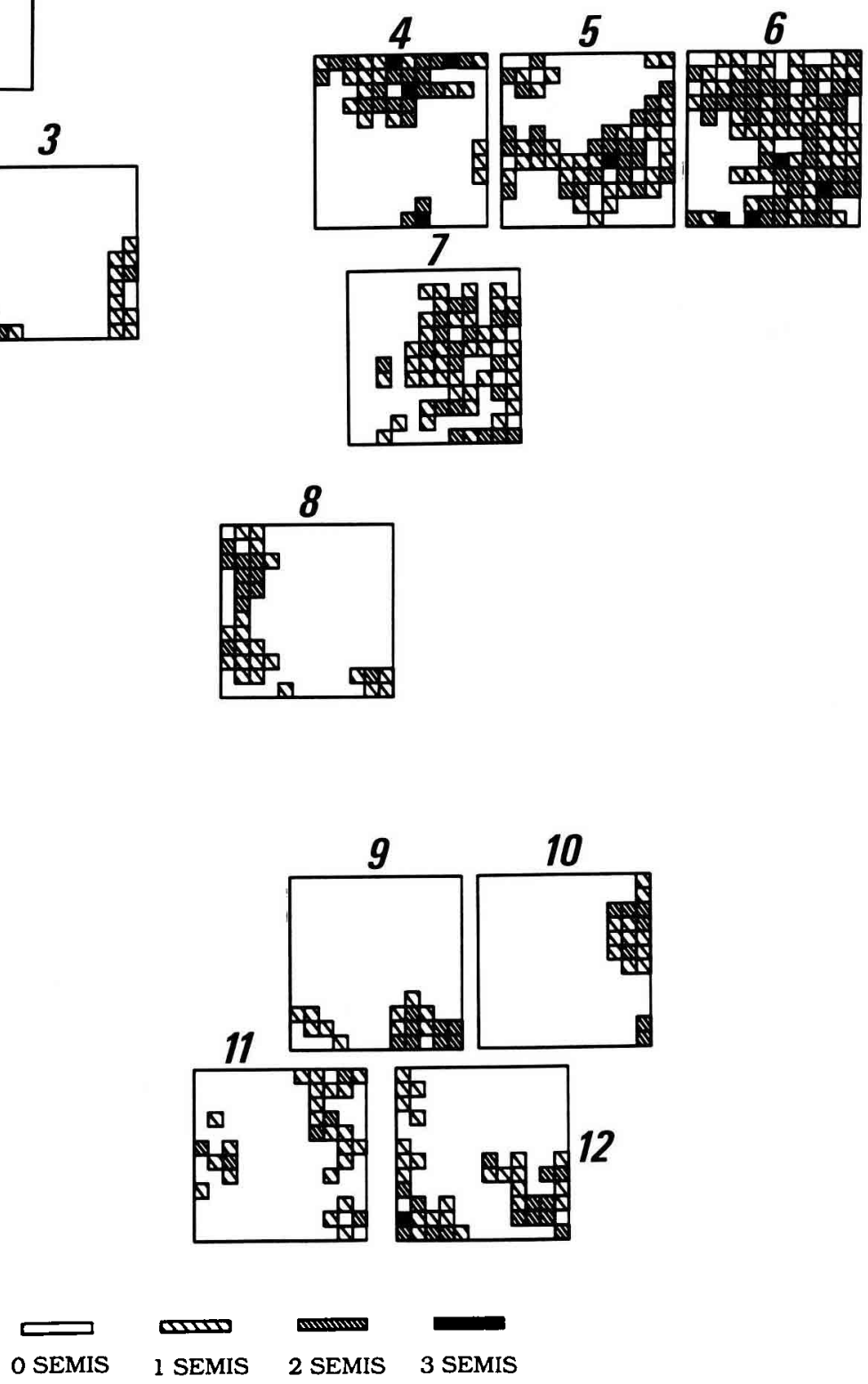

Fig 8. Eperua falcata (petits semis parcelles 1 à 12, inventaire 1986). 


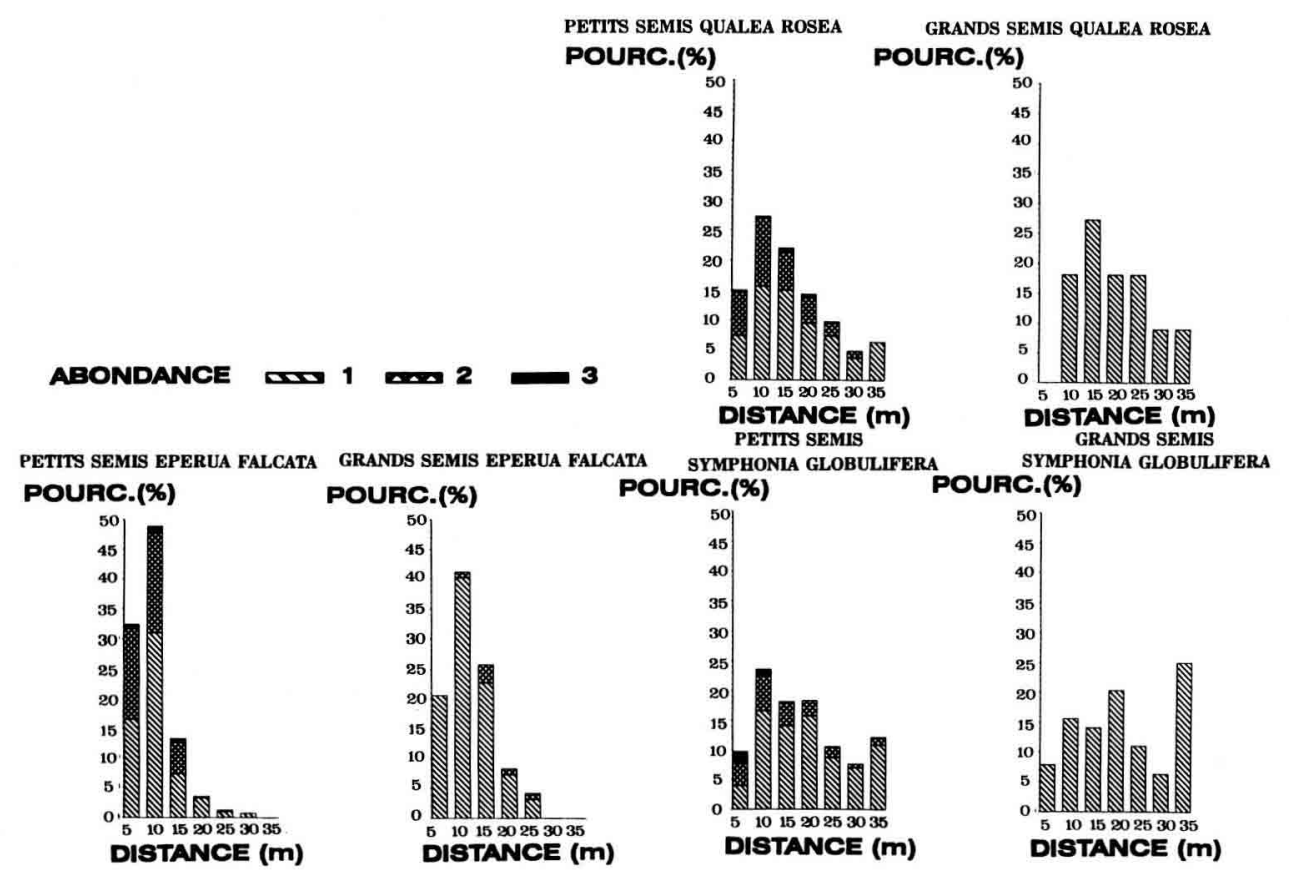

Fig 9. Répartition des petits semis et des grands semis pour les 3 espèces étudiées. Distance : distance du point d'inventaire à l'arbre adulte (diamètre supérieur à $30 \mathrm{~cm}$ ) le plus proche. Abondance : note de présence/abondance. Pourc : fréquence relative observée pour chaque classe d'abondance et de distance (pour $12 \times 144=1728$ relevés).

semblablement à se régénérer sous ellemême en maintenant une structure en "bouquets", mais la dissémination à distance (graines légères et ailées) autorise également une dynamique «centrifuge» favorisant sa survie en ambiance de compétition forte. La cartographie met en évidence cette relation étroite entre semis et semenciers (fig 10).

\section{Eperua falcata}

Pour cette espèce, on ne dispose que d'un inventaire des "wapa» adultes (Eperua falcata et Eperua grandiflora). Cependant $94 \%$ des petits semis sont à moins de $15 \mathrm{~m}$ d'un adulte : dans le cas d'Eperua falcata, espèce autochore et très fructi- fère, la distance au plus proche adulte correspond dans la plupart des cas à la distance au semencier. Comme dans le cas de Qualea rosea, les régénérations sont donc très liées aux arbres du peuplement. Les «plaques» de semis correspondent à une répartition du même type des arbres adultes déjà notée par certains auteurs (Cabrera-Gaillard et Gignoux, 1990). La faible dispersion des graines est la conséquence d'une dissémination essentiellement assurée par une autochorie peu efficace (Forget, 1988). Le fruit est une grousse falciforme qui pend au bout d'un flagelle de 1 à $2 \mathrm{~m}$ de long. La déhiscence est explosive et se produit par temps sec (petite saison sèche). Les graines sont assez lourdes (une dizaine de grammes). 


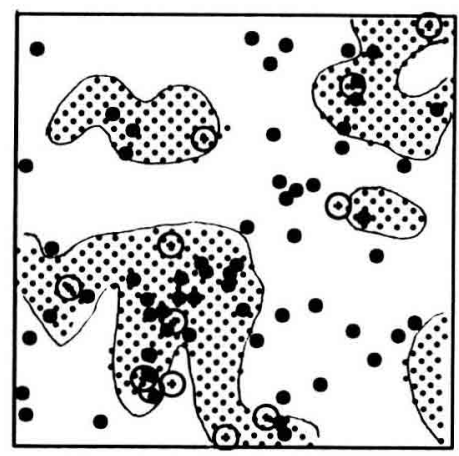

QUALEA ROSEA - PARCELLE 11 (AGREGATS)
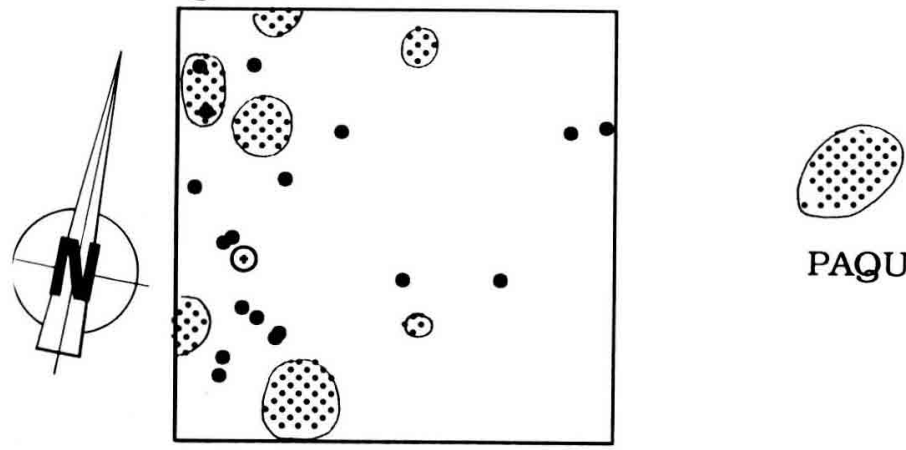

PAQUETS ET AGREGATS

SYMPHONIA GLOBULIFERA - PARCELLE 6 (PAQUETS)
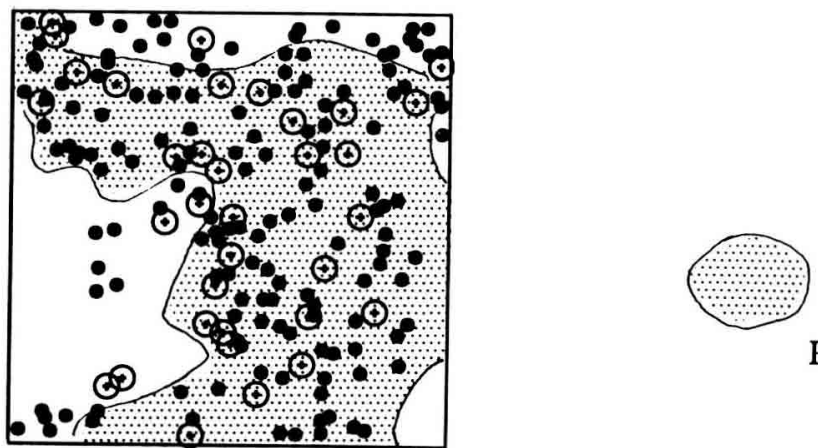

PLAQUE

EPERUA FALCATA - PARCELLE 6 (PLAQUE)

- ARBRES DE DLAMETRE SUPERIEUR A $30 \mathrm{CM}$

○ ARBRES DE DIAMETRE SUPERIEUR A $60 \mathrm{CM}$

Fig 10. Structures des régénérations naturelles et répartition des arbres adultes. 
Forget note que près de $60 \%$ d'entre elles sont projetées à moins de $10 \mathrm{~m}$ du semencier, soit sous la couronne, ce qui rejoint nos propres observations. La sélection semble plus forte à proximité des adultes : les effectifs cumulés pour les distances supérieures à $15 \mathrm{~m}$ représentent $20 \%$ du total pour les petits semis et $38 \%$ pour les grands semis. Les classes d'abondance supérieures à 1 n'apparaissent pratiquement plus pour les grands semis dans un rayon inférieur à $10 \mathrm{~m}$ du "semencier". Ce phénomène de mise à distance a déjà été observé pour cette espèce par d'autres auteurs (Sop Fonkoua, 1988; Forget, 1988) : il correspond d'après la théorie de JanzenConnell à un mécanisme nécessaire pour expliquer le maintien de la diversité dans les forêts tropicales et il suppose, d'après ces mêmes auteurs, l'action de prédateurs spécifiques à proximité des semenciers ou dans les taches de régénération les plus denses (Janzen, 1970; Connell, 1971). Ces hypothèses de prédation n'ont pas été confirmées par Forget qui suspecte, pour Eperua falcata, une interception plus intense de la lumière sous le couvert des arbres adultes. Des travaux récents effectués à Paracou par la Station de recherches forestières de I'INRA, montrent que la répartition de la lumière ne serait pas en cause (HUC, non publié), mais que les semis d'Eperua falcata situés sous les houppiers d'arbres de cette même espèce ont des folioles nécrosés et manifestement une baisse de l'activité photosynthétique par rapport aux témoins situés dans d'autres conditions (Mondy, 1990). Des phénomènes de nature allélopathique pourraient en être la cause; l'existence de substances phytotoxiques a déjà été révélée dans les feuilles fraîches de wapa à l'aide d'une plante test, le cresson alénois (Bariteau, à paraître).

Finalement, l'autochorie, qui impose une dynamique "centripète», est compensée par une mortalité élevée des régénérations à proximité des arbres adultes. Le taux de mortalité des régénérations sur 22 mois est de $60 \%$. Ces caractéristiques, alliées à une grande tolérance vis-à-vis des sols permettent en partie d'expliquer les structures en plaques détectées par le calcul : densités importantes des semis sur les zones «à contraintes élevées", où l'espèce se cantonnerait en raison d'une forte sensibilité à la compétition (Bariteau et al, 1990; Barthes, 1988). Au sein de la plaque, la seule échelle d'agrégation concerne la proximité des semenciers fructifères. Comme dans le cas de Qualea Rosea, la cartographie met en évidence les liaisons étroites entre semis et semenciers (fig 10).

\section{Symphonia globulifera}

$51 \%$ seulement des petits semis se trouvent à moins de $15 \mathrm{~m}$ d'un adulte et $38 \%$ des grands semis. Pour cette dernière catégorie, c'est plus de $25 \%$ de l'effectif qui est dispersé à plus de $30 \mathrm{~m}$ d'un arbre adulte. Cette espèce est classée comme zoochore à grosses graines (volume > $3000 \mathrm{~mm}^{3}$ ) par Charles-Dominique et al (1981). La dissémination est assurée par les rongeurs et les chauves-souris (Forget, 1988). La mortalité entre les stades "petit semis" et "grand semis" est élevée car les classes d'abondance 2 et 3 disparaissent et elle sanctionne moins sévèrement les régénérations éloignées des arbres adultes, comme dans le cas d'Eperua falcata. Le taux de mortalité global à 22 mois n'est cependant que de $22 \%$. La structure en "paquets" observée est en relation avec des pics intenses de fructification à proximité de certains semenciers, et non pas directement sous les arbres fructifères. Les graines sont transportées par les animaux : dans ce cas, il peut y avoir mise en réserve par enfouissement ce qui pourrait expliquer l'existence des "paquets". Ce type de dissémination a été observé par Vouacapoua americana (For- 
get, 1988). Une mortalité sélective accentue cette dynamique "centrifuge". Ce phénomène avait déjà été noté (Bertrand, 1987) : la probabilité d'inventorier la présence en régénération de Symphonia globulifera sur une placette augmente avec une probabilité croissante d'absence de semencier dans un voisinage de $400 \mathrm{~m}^{2}$. Cette constatation est confirmée par la juxtaposition des semenciers et des paquets de semis sur une cartographie (fig 10). La même constatation est faite pour le kimboto (Pradosia cochlearia). Cette répartition des semis est la conséquence directe de la zoochorie, puisque les rongeurs responsables de la dispersion sont aussi des consommateurs des graines. En termes finalistes, cette stratégie serait nécessaire pour assurer des échanges de gènes limitant la dérive génétique et empêchant la création de "cercles de consanguinité". L'image résultante de ce type de dispersion est constituée par un réseau anastomosé, à faible densité ponctué par des pics intenses de régénération.

\section{Présence de semis et facteurs du milieu}

Eperua falcata est la seule espèce étudiée (en ambiance de forêt naturelle) qui présente un indice de fréquence corrigée croissant vers les classes de couvert dominant faible (fig 11). À l'inverse, Eschweilera odora, Bocoa prouacensis, Pradosia cochlearia, Iryanthera sagotiana, Licania spp + Parinari spp, et Moronobea coccinea montrent un comportement d'essences sciaphiles. La même remarque peut être faite pour la topographie (pour respecter un gradient de drainage théorique les classes ont été rangées dans l'ordre : $0,1,2,4,3,5$,). L'abondance relative de Eperua falcata diminue cependant légèrement à proximité des zones les plus basses (cours d'eau temporaires). Sym- phonia globulifera est plus abondant dans les zones inondées ou inondables, de même que Carapa guianensis.

En comparant le nombre de placettes sans régénération d'Eperua falcata et le nombre de placettes où l'étage dominant est absent ou très faible (voir tableau V), on s'aperçoit que les parcelles 5,6 et 7 sont caractérisées à la fois par une abondance des régénérations mais aussi par un étage dominant restreint (les chablis étaient notoirement plus abondants dans ces parcelles en 1986). La mise en comparaison des cartes obtenues sur la régénération de cette espèce et sur le peuplement adulte montre une coïncidence presque absolue (fig 10). La présence de semenciers et de régénération d'Eperua falcata ainsi que la fréquence élevée des chablis sont donc des phénomènes simultanés à Paracou qui induisent une typologie nette des parcelles. Les études menées par Barthes (1988) confirment cette constatation et apportent une explication en terme de pédologie : les zones à horizon imperméable peu profond défavorisent l'enracinement des arbres en le rendant très superficiel. En saison des pluies, les sols gorgés d'eau dans ces zones sont très propices aux chablis. Eperua falcata semble bien adapté à ces conditions, non seulement par ses caractéristiques écophysiologiques, grande plasticité vis-à-vis des facteurs eau et lumière (Bariteau et al, 1990), mais aussi dynamiques : la régénération naturelle est abondante et précoce (constatée souvent sous des arbres de faible diamètre).

D'autres espèces comme Bocoa prouancensis, Pradosia cochlearia et les gaulettes (Licania sp, Parinari sp) apparaissent au contraire inféodés à des stations mieux drainées sur lesquelles la succession «normale» des cycles sylvigénétiques conduit, en théorie, à une fréquence élevée d'espèces "climaciques" et «sciaphiles". 


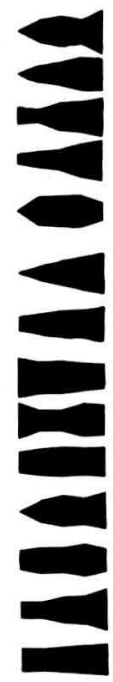

01234

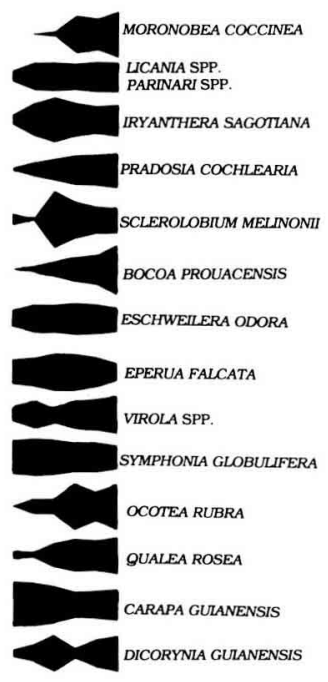

012435
Fig 11. Profils écologiques. Couvert de l'étage dominant (tiges de diamètre supérieur à 10 $\mathrm{cm}$ ) : 0 absent ou faible; 1 couvert inférieur à $20 \%$; 2 couvert compris entre $20 \%$ et $50 \%$; 3 couvert compris entre $50 \%$ et $75 \% ; 4$ couvert supérieur à $75 \%$; (cimes enchevêtrées et feuillage dense). Topographie : 0 crique ou bord de crique, zone inondée ou inondable; 1 marécage; 2 bas de pente, rupture de pente, mais zone non inondable, 3 mi-pente ou haut de pente; 4 replat, rupture de pente; 5 sommet.

\section{CONCLUSION}

Les qualitatifs adoptés par les structures spatiales, dans la terminologie développée par Chessel, sont en rapport étroit avec l'échelle d'observation. Avec des points d'inventaire plus rapprochés, les "paquets» de régénération naturelle de Symphonia globulifera deviendraient des "agrégats». De même la notion de "plaque» pour Eperua falcata prend tout son sens avec la cartographie de la totalité du dispositif (fig 8). Les modèles de répartition spatiale ne sont donc définis que pour une échelle donnée, et dans le cas d'un échantillonage systématique, pour une maille d'observation fixée.

Pour toutes les espèces inventoriées en régénération naturelle à Paracou, l'indice de dispersion calculé au niveau de la maille élémentaire indique une répartition agrégative au sens large, c'est-à-dire jamais conforme à la loi de Poisson ni régulière. Cela rejoint les constatations de nombreux auteurs ayant étudié la répartition en "peau de panthère" des espèces de la forêt tropicale humide (Webb et al, 1972). Les structures observées dans les trois cas étudiés sont en rapport avec le mode de dissémination des graines.

L'anémochorie, peu répandue et peu efficace dans ce type de forêt correspond, dans le cas étudié, à une structure en agré-

Tableau V. Relations entre couverture de l'étage dominant et présence d'Eperua falcata en régénération à Paracou avant exploitation. En italique : pourcentage de placettes pourvues en Eperua falcata.

$\begin{array}{lllllllllllll}\text { Numéros de parcelles } & 1 & 2 & 3 & 4 & 5 & 6 & 7 & 8 & 9 & 10 & 11 & 12\end{array}$

Nombre des placettes vides d'Eperua falcata

Nombre de placettes avec et. dominant note 0 ou 1

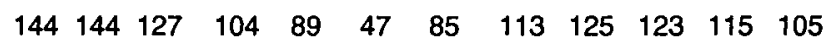

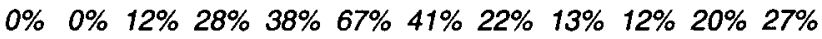

$\begin{array}{llllllllllll}8 & 17 & 15 & 21 & 26 & 22 & 33 & 10 & 6 & 3 & 6 & 6\end{array}$


gats. Ce mode de dispersion est celui de $7 \%$ des espèces de la forêt guyanaise (Riera et al, 1990). La dimension des agrégats de régénération, dans le cas de Qualea rosea, ont la taille d'un quart de parcelle (1 à 1,5 ha). Une répartition en «bouquets", tant au niveau des régénérations que des arbres adultes, a déjà été décrite pour une autre espèce à dissémination anémochore, Dicorynia guianensis (Forget, 1988; Cabrera et Gignoux, 1990). Le mode de régénération de cette espèce est caractérisé, d'après Forget, par la dormance de certaines graines (création d'un potentiel séminal édaphique) et par une tolérance à l'ombre des stades juvéniles («potentiel végétatif»). En l'absence de ces caractéristiques, qui permettent à la régénération d'attendre une mise en lumière favorable à sa survie et à sa croissance, la pérennité de l'espèce serait menacée.

L'autochorie est également peu répandue en forêt tropicale humide de Guyane ( $5 \%$ des espèces d'après Riera), mais elle joue cependant un rôle très important en raison de la grande abondance d'Eperua falcata dans les peuplements. La présence de plaques résulte de la faible dispersion des graines qui est compensée par une mortalité sélective au profit des plantules les plus éloignées des arbres adultes. Une action de nature allélopathique est suspectée et étudiée. Ce type d'interaction entre strates, voire entre espèces pourrait expliquer partiellement le maintien de la diversité spécifique en forêt tropicale humide (Clark et Clark, 1984). De plus, l'hétérogénéité des sols du Bouclier Guyanais induit une différentiation spatiale des peuplements en pratiquant une ségrégation des espèces : les milieux "pauvres" sont caractérisés par des peuplements à taux de renouvellement rapide; les espèces qui prospèrent dans de tels sites sont par nature peu exigeantes sur le plan trophique, plastiques, adaptées aux ouvertures brutales du couvert, et doivent fructifier précocement. Eperua falcata est une légumineuse qui répond parfaitement à ces critères. Au Surinam, elle est décrite comme dominante dans des formations xérophiles sur sable blanc (Schultz, 1960). En Guyane, dans la forêt de la bande littorale sur socle, elle domine sur les sols à drainage vertical bloqué. Sa présence permet donc en théorie, dans cette dernière zone, de cartographier les sols «à contraintes fortes».

La zoochorie est le mode classique de dispersion des graines en forêt guyanaise : plus de $80 \%$ des espèces sont zoochores (Riera et al, 1990). Les caractéristiques de la dissémination sont alors en relation avec le comportement des animaux qui effectuent le transport. C'est la taille des graines qui détermine le type des vertébrés frugivores assurant la dispersion (Charles-Dominique et al, 1981). Les structures spatiales observées en forêt tropicale humide, aussi bien au niveau des régénérations que des peuplements adultes qui en sont issus, sont dans la plupart des cas la conséquence du "mutualisme» existant entre végétaux et animaux. La dissémination à grande distance constitue, dans ce cas, un élément fondamental de la régénération naturelle, qui ne peut pas, sans dispersion efficace des graines, trouver les conditions favorables à sa survie et à sa croissance (Riera et al, 1990).

L'inventaire systématique des régénérations naturelles entrepris sur le site de $\mathrm{Pa}$ racou en Guyane française permettra d'apporter d'ici une quinzaine d'années un verdict sur la pertinence des traitements sylvicoles en terme de pérennité de l'écosystème. Les contraintes pratiques et la complexité du milieu limitent la portée des études entreprises. Mais la méthodologie proposée permet déjà de construire, pour quelques espèces bien représentées, des modèles de dynamique spatiale en liaison avec leurs caractéristiques biologiques. 
La dynamique de la forêt à Paracou représente un cas particulier par rapport au schéma classique des cycles sylvigénétiques rencontrés dans d'autres types de forêt en Guyane (Oldeman, 1974). À l'échelle d'une parcelle $(6,25 \mathrm{ha})$, il a été mis en évidence des typologies forestières directement liées à l'hétérogénéité du substrat : les sols à horizons imperméables à faible profondeur portent des peuplements à renouvellement rapide. La forêt "climacique" n'apparaît pas dans ces conditions.

La sylviculture doit largement tenir compte de ces conclusions : il he suffit pas d'intervenir sur les niveaux d'éclairement au sol par coupes ou éclaircies pour modifier les processus de régénération. Des interactions importantes entre strates ont été mises en évidence : le rôle de la litière et des phénomènes de nature allélopathique seraient à prendre en compte, en plus de l'influence de la lumière et de la faune, pour mieux comprendre la dynamique des populations gérées. Cela implique une grande prudence dans le suivi des expérimentations sylvicoles, en particulier dans l'étude de l'impact des phytocides sur le milieu. Un des traitements sylvicoles appliqué à Paracou utilise en effet des techniques de dévitalisation chimique. L'augmentation de la pression de chasse, conséquence de l'ouverture des pistes de pénétration, risque également de modifier indirectement les conditions de régénération en modifiant la faune présente.

Enfin, la compréhension des mécanismes de sylvigenèse passe désormais, plus que par une unification des "théories", par des outils de modélisation performants capables d'intégrer un grand nombre de processus biologiques dont la connaissance est progressivement acquise. Les taux de renouvellement des peuplements et les probabilités conditionnelles de survie des semis font sans aucun doute partie des données de base devant servir "d'entrées" aux modèles.

\section{REMERCIEMENTS}

Cette étude a pu être réalisée grâce au financement de la Région Guyane. Que soient aussi remerciés les organismes, dont le personnel et les chercheurs travaillent en coopération avec I'INRA sur le projet Paracou, et particulièrement le Centre technique forestier tropical fondateur du dispositif sylvicole. Merci au professeur $H$ Puig, de l'université Paris VI, pour ses conseils pertinents et indispensables en matière de botanique tropicale. Merci à Laurent Lapchin de I'INRA d'Antibes pour son logiciel de calcul des indices non paramétriques.

\section{RÉFÉRENCES}

Bachacou J, Decourt N (1976) Étude de la compétition dans des plantations régulières à l'aide de variogramme. Ann Sci For 33(4), 177-198

Bariteau M, Geoffroy J (1989) Sylviculture et régénération naturelle en forêt guyanaise. Rev For Fr XLI (4), 309-323

Bariteau M, Huc R, Montpied P (1990) Évaluation de l'impact de traitements sylvicoles en forêt tropicale humide sur la régénération; illustration à partir du comportement in situ et en conditions contrôlées de deux espèces de Guyane. Atelier sur l'aménagement et la conservation de l'écosystème forestier tropical humide, Cayenne, mars 1990, étude de cas, $10 \mathrm{p}$

Barnard RC (1950) Linear regeneration sampling. Malay For XIII, 3, p 129, sqq

Barthes B (1988) Ou pousse cet arbre ? Premiers résultats à l'étude des relations solvégétaux en forêt de Paracou-Guyane. Rapport interne Orstom-CTFT, $33 \mathrm{p}$

Bertrand C (1987) Contribution à l'étude de l'impact des traitements sylvicoles sur la régénération naturelle en Guyane. Mise au point d'une méthodologie d'inventaire et premières observations avant l'exploitation forestière. Mémoire ESAT, $67 p$

Bouchon J (1979) Structure des peuplements forestiers. Ann Sci For 36(3), 175-209

Cabrera-Gaillard C, Gignoux J (1990) Répartitions spatiales et sylviculture en forêt guyanaise. Atelier sur l'aménagement et la conservation de l'écosystème forestier tropical 
humide, Cayenne, mars 1990, études de cas; $19 p$

Charles-Dominique $P$, Atramentowicz $M$, Charles Dominique $M$, Gérard $H$, Hladik $A$, Hladik CM, Prévost MF (1981) Les mammifères frugivores arboricoles nocturnes d'une forêt guyanaise : interrelations plantesanimaux. Rev Écol Terre Vie 35, 342-435

Chessel D (1978) Description non paramétrique de la dispersion spatiale des individus d'une espèce. In: Biométrie et écologio (JM Legay, $R$ Tomassone, eds) Société française de biomé-trie, $n^{\circ} 1,45-136$

Clark DA, Clark DB (1984) Spacing dynamics of a tropical rain forest tree: evaluation of the Janzen-Connel model. Am Nat 124, 769-788

Connell JH (1971) On the role of natural enemies in preventing competitive exclusion in some marine animals and in rain forest trees. In: Dynamics of populations (PJ den Boer, GR Gradwell, eds) Proc Adv Study Inst Dynamics of numbers in population. Oosterbeek, 1970. Centre for Agricultural Publish-ing and Documentation, Wageningen, 298-310

Daget P, Delpech R, Possonnet J, Romane F (1974) Approche méthodologique plus particulièrement appliquée aux prairies. In: Contributions de la phytoécologie au diagnostic des conditions hydriques des sols. BTI 271-272, 759-773

Dagnélie $P(1970)$ Théorie et méthodes statistiques. Vol 2. Les Presses Agronomiques de Gembloux, Gembloux, 463 p

Diggle PJ (1983) Statistical analysis of spatial point pattems. Academic Press, London, $148 \mathrm{p}$

Forget PM (1988) Dissémination et régénération naturelle de huit espèces d'arbres en forêt guyanaise. Thèse, Univ Paris VI, $245 \mathrm{p}$

Hladik A (1979) Les variables régionalisées dans l'interface végétal-primate : étude appliquée à des espèces arborescentes d'une forêt semi-décidue du Sri Lanka. Ecol Plant 14 (3), 273-287

Janzen DH (1970) Herbivores and the number of tree species in tropical forests. Am Nat 104, 501-528
Matheron G (1970) La théorie des variables régionalisées et ses applications. Les cahiers du centre de morphologie mathématique de Fontainebleau. Fasc 5, École nationale supérieure des Mines, Paris, $212 p$

Maitre HF (1982) Projet de recherches sylvicoles sur les peuplements naturels en forêt dense guyanaise. Centre technique forestier tropical, Nogent-sur-Marne, $65 p$

Mondy N (1990) Étude des phénomènes d'allélopathie chez Eperua falcata Aublet. Mémoire Enfa, $36 \mathrm{p}$

Oldeman RAA (1974) L'architecture de la forêt guyanaise. Mémoire Orstom 73, Paris, $204 p$

Riera B, Puig H, Lescure JP (1990) La dynamique de la forêt naturelle. Bois For Trop, spécial Guyane $n^{\circ} 219,69-78$

Rollet $B$ (1969) La régénération en forêt dense humide sempervirente de plaine de la Guyane vénézuélienne. Bois For Trop 124, 19-38

Rosevear DR, Lancaster PC (1953) Historique et aspect actuel de la sylviculture en Nigeria. Bois For Trop 28, 3-12

Schmitt L, Bariteau M (1990) Gestion de l'écosystème forestier guyanais. Étude de la croissance et de la régénération naturelle. Dispositif de Paracou, Bois For Trop $n^{\circ} 220$ spécial Guyane, 3-23

Schultz JP (1960) Ecological studies in rain forest in northern Suriname. Verh $K$ Ned Akad Wet Afd Natuurk D Tweede Reeks 2, 53 (1), 1-267

Sop Fonkoua D (1988) Contribution à une étude de mortalité des régénérations naturelies en forêt dense guyanaise. Mémoire Esat, $40 \mathrm{p}$

Upton GJG, Fingleton B (1985) Spatial data analysis by example. Vol I: Point pattern and quantitative data. Wiley, Chichester, $150 \mathrm{p}$

Webb LJ, Tracey JG, Williams WT (1972) Regeneration and pattern in the subtropical rain forest. $J$ Ecol $60,675-695$

Wyatt-Smith $J$ (1963) Manual of Malayan silviculture for inland forests. Vol 1. Malay For Rec 23, Forest Department, Malaya 